\title{
COVID-19 induced immune thrombocytopenic purpura: case report
}

\author{
Sara Ashraf, Mohamed Alsharedi \\ Joan C Edwards School of Medicine at Marshall University, Huntington, WV, USA \\ Correspondence to: Sara Ashraf. Joan C Edwards School of Medicine at Marshall University, Huntington, WV, USA. Email: saraashraf87@yahoo.com.
}

\begin{abstract}
Immune thrombocytopenic purpura (ITP) is an autoimmune state of decreased platelets caused by antibody or T-cell mediated destruction of platelets through the reticuloendothelial system and impairment of their production. Symptoms of ITP include bleeding usually from nose or gums, easy bruising, petechiae commonly of lower extremities, menorrhagia, hematuria, hematemesis, hematochezia and most dreadful, intra cranial hemorrhage. Molecular mimicry between viral antigens and host platelet antigens forming cross-reactive anti-platelet autoantibodies may lead to increased platelet clearance in ITP associated with viral infections. One of the many viruses associated with this is the Coronavirus disease 2019 (COVID-19). It has caused a devastating pandemic. It can activate innate and adaptive immune responses. It has numerous signs and symptoms including but not limited to dyspnea, fever, cough, fatigue, myalgias, loss of taste and smell. It leads to diseases such as pneumonia, acute respiratory distress syndrome, thrombosis and cardiomyopathy. Hematologic manifestations include thrombocytopenia and more commonly lymphopenia. Treatment includes steroids, immune globulin, romiplostim, eltrombopag, rituximab or splenectomy. Contact sports should be avoided due to risk of intra cranial bleeding with head impact. Nonsteroidal anti-inflammatory drugs (NSAIDs) and aspirin should be used with caution since they impair platelet function. We discuss a patient with COVID-19 who developed thrombocytopenia thought to be due to ITP. Not much is known about the association between the two. It is important to keep this differential in mind when taking care of patients with COVID-19 who develop thrombocytopenia.
\end{abstract}

Keywords: Coronavirus disease 2019 (COVID-19); immune thrombocytopenic purpura (ITP); platelets; intravenous immune globulin (IVIG); case report

Received: 23 October 2020; Accepted: 29 April 2021; Published: 09 July 2021.

doi: $10.21037 /$ sci-2020-060

View this article at: http://dx.doi.org/10.21037/sci-2020-060

\section{Case presentation}

A 62-year-old morbidly obese female with past medical history significant for pulmonary hypertension and hypothyroidism presented with a one-week history of worsening shortness of breath, non-productive cough and subjective fevers.

Patient's brother had tested positive for COVID-19 one week before her symptoms had developed. Our patient was hospitalized with respiratory distress. The patient was afebrile, pulse of 82 beats per minute, 28 breaths per minute with oxygen saturation 90 percent on high flow oxygen by nasal cannula at 15 liters. Physical examination was significant for decreased breath sounds.

Complete blood count on admission showed white blood cell count of 3.6 per cubic millimeter, absolute lymphocyte count of 0.3 ; hemoglobin of $13.6 \mathrm{~g}$ per deciliter, and platelet count of 76,000 per cubic millimeter. She had a normal platelet count noted previously in the years past.

Chest X-ray on admission showed no acute abnormality. The patient had a rapid nasal swab COVID-19 testing and was positive on the day of admission. She was started on hydroxychloroquine. However, on follow up EKG's there was a prolonged QTc interval and accordingly hydroxychloroquine was stopped on the fourth day of hospitalization. She was then started on Doxycycline. 
Doxycycline was stopped after 7 days (day 11).

Patient continued to report shortness of breath during the hospitalization. On the sixth day of hospitalization, repeat chest $\mathrm{X}$-ray showed extensive bilateral pulmonary opacities. She had worsening oxygen requirements and had to be placed on heated humidified high flow nasal cannula (Opti-Flow) with $\mathrm{FiO}_{2}$ 100\%, her saturations improved.

On day 12, the patient started to improve and oxygen requirements decreased. Repeat chest X-ray and laboratory parameters were consistent with improvement as c-reactive protein and d-dimer were trending down. CBC also had improved with platelet count of 171,000 per cubic millimeter and an absolute lymphocyte count of 0.9 .

Despite the overall improvement, on day 14, the patients' labs showed a down trending platelet count with no other cytopenia. Platelet count fell to 102,000 per cubic millimeter, then to $44,000,33,000,12,000,24,000$ and then 14,000 . It was noticed that mean platelet volume (MPV) had increased from 8.7 to 11.4; while white blood cell count and hemoglobin had remained stable. Clinically the patient had no bleeding, petechiae or bruising.

The patient was on prophylactic dose of low molecular weight heparin since hospitalization, which was discontinued when the platelet count had reached 44,000 per cubic millimeter.

On day 17 , as the platelets were down to 24,000 per cubic millimeter with no apparent cause, hematology service was consulted for evaluation. Work-up was initiated to rule out secondary causes. Peripheral blood smear showed no platelet clumping but revealed giant and large forms. HIT was unlikely with low 4T score of 2 and given the patient was on low molecular weight heparin and not unfractionated heparin. Disseminated intravascular coagulation panel testing was unremarkable with normal values of PT, PTT and fibrinogen. Hepatitis and HIV testing were negative.

Results were suggestive of immune related thrombocytopeniaITP. Accordingly, we started treatment with infusion of intravenous immune globulin (IVIG) 1 gram per kilogram. This was well tolerated. Platelets increased from 14,000 per cubic millimeter to 68,000 per cubic millimeter, supporting our diagnosis of ITP in the setting of COVID-19. One week later, the patient's platelet count normalized at 185,000. We had refrained from giving the patient high dose steroids or dexamethasone challenge due to initial recommendations of avoiding it in COVID-19.

All procedures performed in studies involving human participants were in accordance with the ethical standards of the institutional and/or national research committee(s) and with the Helsinki Declaration (as revised in 2013). Our institutional policy is to waive IRB approval as there is no personal information disclosed, no experimental treatment involved and patient consent was obtained.

\section{Discussion}

ITP is an autoimmune disorder, characterized by isolated thrombocytopenia, where dysregulation of the immune system results in antibodies directed against platelet glycoprotein IIb/IIIa complex, causing platelet destruction through the reticuloendothelial system and impairment of platelet production (1). It can be primary and occur de novo, or secondary in the setting of an infection or other autoimmune disorders (1).

Annual incidence of ITP in the US was 6.1 per 100,000 persons, more commonly found in females than males like other autoimmune disorders. The prevalence in adults is highest amongst elderly patients, greater than or equal to 65 years of age (2). ITP can be clinically classified into 3 phases. The first phase is called newly diagnosed and occurs within the first 3 months. The second phase is persistent ITP lasting between 3 and 12 months. The third phase is termed chronic ITP, in which symptoms last for more than 12 months. The first line treatments work inhibiting autoantibody production and platelet degradation, second line treatments are based on immunosuppression, such as Rituximab, and splenectomy and third line treatments focus on stimulating platelet production by megakaryocytes (3).

ITP has been linked to various viral infections such as hepatitis C, HIV, CMV and influenza, rubella, mumps, varicella, parvovirus, and Epstein-Barr virus (4), however, not much is known about its association with COVID-19. Infectious etiologies of ITP are felt to adapt molecular mimicry. It is a process whereby viral antigen is recognized as being similar to a platelet antigen, giving rise to crossreactive anti-platelet autoantibodies (3).

Thrombocytopenia may be a complication of COVID-19 (5). In a recent meta-analysis, extent of thrombocytopenia was found to be directly proportional to the severity of COVID-19 (5). It is felt to be generally multifactorial (5). The virus can directly infect the bone marrow, causing impairment of hematopoiesis and formation of antibodies against platelets (6-8). The immune system wreaks havoc and destroys platelets and causes hemophagocytosis. Cytokine storm destroys bone marrow 
progenitor cells reducing platelet production. Lung injury entraps megakaryocytes and hinders the release of platelets from megakaryocytes. Platelet aggregation in the lungs leads to microthrombi and platelet consumption (8).

A recently reported case was also felt to be suspicious for ITP in a COVID-19 positive patient (9). Our patient had a previous history of hypothyroidism, perhaps making her more prone to ITP. It will be important for us to see if other patients have similar findings. Our case was interesting in that the patient had platelet recovery and then a delayed acute drop in platelet count despite clinical improvement. She responded well to IVIG, supporting an immune mediated thrombocytopenia.

\section{Acknowledgments}

Funding: None.

\section{Footnote}

Reporting Checklist: The authors have completed the CARE reporting checklist. Available at http://dx.doi.org/10.21037/ sci-2020-060

Conflicts of Interest: Both authors have completed the ICMJE uniform disclosure form (available at http://dx.doi. org/10.21037/sci-2020-060). The authors have no conflicts of interest to declare.

Ethical Statement: The authors are accountable for all aspects of the work in ensuring that questions related to the accuracy or integrity of any part of the work are appropriately investigated and resolved. All procedures performed in studies involving human participants were in accordance with the ethical standards of the institutional and/or national research committee(s) and with the Helsinki Declaration (as revised in 2013). Our institutional policy is to waive IRB approval as there is no personal information disclosed, no experimental treatment involved and patient consent was obtained.

doi: $10.21037 /$ sci-2020-060

Cite this article as: Ashraf S, Alsharedi M. COVID-19 induced immune thrombocytopenic purpura: case report. Stem Cell Investig 2021;8:14.
Open Access Statement: This is an Open Access article distributed in accordance with the Creative Commons Attribution-NonCommercial-NoDerivs 4.0 International License (CC BY-NC-ND 4.0), which permits the noncommercial replication and distribution of the article with the strict proviso that no changes or edits are made and the original work is properly cited (including links to both the formal publication through the relevant DOI and the license). See: https://creativecommons.org/licenses/by-nc-nd/4.0/.

\section{References}

1. Johnsen J. Pathogenesis in immune thrombocytopenia: new insights. Hematology Am Soc Hematol Educ Program 2012;2012:306-12.

2. Weycker D, Hanau A, Hatfield M, et al. Primary immune thrombocytopenia in US clinical practice: incidence and healthcare burden in first 12 months following diagnosis. J Med Econ 2020;23:184-92.

3. Zufferey A, Kapur R, Semple JW. Pathogenesis and Therapeutic Mechanisms in Immune Thrombocytopenia (ITP). J Clin Med 2017;6:16.

4. Cines DB, Bussel JB, Liebman HA, et al. The ITP syndrome: pathogenic and clinical diversity. Blood 2009;113:6511-21

5. Lippi G, Plebani M, Henry BM. Thrombocytopenia is associated with severe coronavirus disease 2019 (COVID-19) infections: A meta-analysis. Clin Chim Acta 2020;506:145-8.

6. Yang M, Ng MH, Li CK. Thrombocytopenia in patients with severe acute respiratory syndrome (review). Hematology 2005;10:101-5.

7. Jolicoeur P, Lamontagne L. Impairment of bone marrow pre-B and B cells in MHV3 chronically-infected mice. Adv Exp Med Biol 1995;380:193-5.

8. Xu P, Zhou Q, Xu J. Mechanism of thrombocytopenia in COVID-19 patients. Ann Hematol 2020;99:1205-8.

9. Zulfiqar AA, Lorenzo-Villalba N, Hassler P, et al. Immune Thrombocytopenic Purpura in a Patient with Covid-19. N Engl J Med 2020;382:e43. 